\title{
Effect of phosphorus application rate on Mentha spicata L. grown in deep flow technique (DFT)
}

\author{
Antonios Chrysargyris ${ }^{\mathrm{a}}$, Spyridon A. Petropoulos ${ }^{\mathrm{b}}$, Ângela Fernandes ${ }^{\mathrm{c}}$, Lillian Barros ${ }^{\mathrm{c}}$, \\ Nikolaos Tzortzakis ${ }^{\mathrm{a}, *}$, Isabel C.F.R. Ferreira ${ }^{\mathrm{c}, *}$

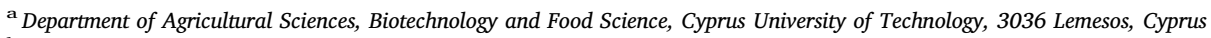 \\ ${ }^{\mathrm{b}}$ Laboratory of Vegetable Production, University of Thessaly, Fytokou Street, 38446 N. Ionia, Magnissia, Greece \\ ' Centro de Investigação de Montanha (CIMO), Instituto Politécnico de Bragança, Campus de Santa Apolónia, 5300-253 Bragança, Portugal
}

\section{A R T I C L E I N F O}

\section{Keywords:}

Essential oil

Mentha spicata L.

Organic acids

Phenolic compounds

Phosphorus

Spearmint

\begin{abstract}
A B S T R A C T
The present study evaluated the impact of phosphorus application rate on plant growth and physiological parameters, antioxidant activity, chemical composition, and essential oil yield and composition of hydroponically grown spearmint plants. Increased $\mathrm{P}$ levels resulted in high dry matter content of the aerial part. Antioxidant activity of spearmint leaves was significantly higher at the highest P levels. Although essential oil yield was not affected, essential oil composition varied among the studied P levels, especially carvone content. Total and individual organic acids content was higher when $50 \mathrm{mg} / \mathrm{L} \mathrm{P}$ were added in the nutrient solution. Rosmarinic acid was the main detected phenolic compound, while the highest total phenolic compounds and rosmarinic acid content was observed at 50 and $70 \mathrm{mg} / \mathrm{L}$ of $\mathrm{P}$, respectively. In conclusion, phosphorus application rate may affect spearmint growth and development, as well as chemical composition and essential oil composition.
\end{abstract}

\section{Introduction}

Phosphorus (P) uptake from plants has been suggested to determine photosynthetic potential, while any deficiencies are associated with reduced carbon fixation in the chloroplasts. Furthermore, high P application rates have been shown to decrease Matricaria chamomilla L. essential oil yield (Emongor, Chweya, Keya, \& Munavu, 1990), whereas they are associated with increased oil yield of Salvia officinalis L. (Nell et al., 2009). Moreover, interactions between minerals have been usually found to be stronger than the action each individual mineral may have. Research on marjoram (Origanum majorana L.) has reported that increasing phosphorus application rates up to $3 \mathrm{mM}$ resulted in an increase of total volatile oil yield by $50 \%$ (Trivino \& Johnson, 2000). Moreover, in the study of Ramezani, Rezaei, and Sotoudehnia (2009) phosphorus was applied on basil plants (Ocimum basilicum L.) by foliar spray at two growth stages, resulting in a significant increase of essential oil yield, without however affecting fresh and dry weight of the aerial biomass. Considering that phosphorus is generally applied in soil with basal dressing prior to seeding or planting, the effectiveness of phosphorus fertilization on plant growth and essential oil content for soilless growing systems needs further examination.

At present, an increasing interest has been noted both in industrial and scientific research for using compounds and extracts of medicinal and aromatic plants as alternatives to synthetic antioxidants, due to their powerful antimicrobial and antioxidant properties. According to Kivilompolo and Hyötyläinen (2007), spearmint leaves are a rich source of rosmarinic, chlorogenic and caffeic acids. Rita, Pereira, Barros, Santos-Buelga, and Ferreira (2016) suggested that antioxidant activity of infusions from apical leaves of spearmint was due to phenolic compounds content and rosmarinic acid in particular, while Gonçalves et al. (2017) also attributed antioxidant potential of spearmint leaves to other phenolic compounds apart from rosmarinic acid, as well to non-phenolic compounds with synergistic effects.

In soilless production systems the nutrient are supplied through the nutrient solutions enabling rapid growth and biomass production while nutrient solution composition is fully controlled and adjusted according to plant requirements (Garlet \& Santos, 2008). Float cultivation systems are successfully used for the commercial production of various leafy vegetables such as endive, lettuce, radish, rocket, spinach and so forth. These production systems are also very promising for aromatic plants and herbs and micro-greens production. The aim of the present study was to investigate the effect of five different phosphorus application rates $(30-70 \mathrm{mg} / \mathrm{L}$ of nutrient solution) on agronomic performance, mineral composition, and antioxidant properties, as well as on essential

\footnotetext{
* Corresponding authors.

E-mail addresses: nikolaos.tzortzakis@cut.ac.cy (N. Tzortzakis), iferreira@ipb.pt (I.C.F.R. Ferreira).
} 
oil, organic acids and phenolic compounds composition of $M$. spicata plants grown in a deep flow hydroponic system.

\section{Materials and methods}

\subsection{Plant material and growing conditions}

The current study was carried out during the spring-summer period at the experimental greenhouse of the Cyprus University of Technology, Limassol, Cyprus. Air temperature ranged between 17.7 and $29.0^{\circ} \mathrm{C}$ during the experimental period. Plant material and growing conditions have been described by the authors in previous experiments regarding the effect of potassium on spearmint growth and chemical composition (Chrysargyris, Xylia, Botsaris, \& Tzortzakis, 2017). Briefly, spearmint plants were grown from cuttings (obtained from the Cypriot National Centre of Aromatic Plants) which were transplanted in plastic 1-L containers (one plant in each container) when they reached $10 \mathrm{~cm}$ in height. Containers were sorted according to randomized complete blocks design with each replication consisting of a group of 6 pots, while plant density was arranged at 16 plants $/ \mathrm{m}^{2}$. Nutrient solution composition throughout the experimental period is presented in Supplementary Material Table S1, while nutrient solution consumption and $\mathrm{P}$ content in nutrient solution after uptake throughout the growing period are presented in Supplementary material (Figs. S1 and S2, respectively). By measuring the amount of nutrient solution that was added to each container throughout the growing period in order to keep the volume in each container constant $(1 \mathrm{~L})$, consumption could be calculated. The impact of $\mathrm{P}$ levels was examined by applying five levels of $30,40,50,60$ or $70 \mathrm{mg} / \mathrm{L}$ of P (P30, P40, P50, P60 or P70, respectively), while $\mathrm{N}$ and $\mathrm{K}$ content in nutrient solution was kept at $200 \mathrm{mg} / \mathrm{L}$ and $375 \mathrm{mg} / \mathrm{L}$, respectively, based on the results of previous studies by our team (Chrysargyris, Panayiotou, \& Tzortzakis, 2016; Chrysargyris et al., 2017). For each P level there were three replications of six plants (18 plants in total for each treatment). The plants were treated with the above described nutrient solution for five weeks. Nutrient solution was checked every week for nutrients replenishment, while $\mathrm{pH}$ and electrical conductivity (EC) were adjusted every second day at 5.8 and $\mathrm{EC}=2.1 \mathrm{mS} / \mathrm{cm}$, respectively.

\subsection{Plant growth}

Ninety spearmint plants were grown for five weeks and until anthesis took place, treated with the above described P levels (treatments). Detailed measurements for plant growth assessment (aerial part and root fresh and dry plant weight, the number of leaves, plant height, root length, and stem thickness) were obtained from six individual plants for each $\mathrm{P}$ level according to the methods described by Chrysargyris et al. (2017). Briefly, weight was measured with a laboratory scale, plant height and root length were measured at the furthest point with a measuring tape, and stem thickness was measured with a caliper.

\subsection{Physiological parameters}

\subsubsection{Chlorophyll determination}

Chlorophylls content (Chlorophyll $a, b$ and total chlorophylls) was determined according to the method previously described by Richardson et al. (2002). Briefly, stomatal conductance of leaves was measured with the aid of a dynamic porometer (Delta-T AP4; Delta-T Devices-Cambridge, UK), while maximum $\mathrm{F}_{\mathrm{v}} / \mathrm{F}_{\mathrm{m}}$ photochemical quantum yields of PSII were measured with a Chlorophyll Fluorometer (OptiSci OS-30p; Opti-Sciences, Hudson, NH) after incubating leaves for 20 min in dark conditions before recording of measurements.

\subsubsection{Activities of antioxidant enzymes}

Antioxidant enzymes activity (APX, CAT and SOD) was determined according to the methods previously described by the authors. Briefly, extraction of previously homogenized fresh leaf samples was carried out with the use of ice cold extraction buffer ( $1 \mathrm{mM}$ ethylenediaminetetraacetic acid (EDTA), 1\% ( $w / v)$ polyvinylpyrrolidone (PVPP), $1 \mathrm{mM}$ phenylmethylsulfonyl fluoride (PMSF) and $0.05 \%$ Triton X-100 in $50 \mathrm{mM}$ potassium-phosphate buffer $(\mathrm{pH}=7.0)$ ) (Jiang \& Zhang, 2002). Results for APX and CAT were expressed as enzyme units per mg of protein ( 1 unit $=1 \mathrm{mM}$ of $\mathrm{H}_{2} \mathrm{O}_{2}$ reduction/min), while SOD activity was estimated as the amount of enzyme that resulted in 50\% inhibition of the NBT photoreduction rate.

\subsubsection{Determination of $\mathrm{H}_{2} \mathrm{O}_{2}$ content and lipid peroxidation}

$\mathrm{H}_{2} \mathrm{O}_{2}$ content was determined based on the method of Loreto and Velikova (2001), while lipid peroxidation was assessed according to method of De Azevedo Neto, Prisco, Enéas-Filho, Abreu, and GomesFilho (2006). $\mathrm{H}_{2} \mathrm{O}_{2}$ content was expressed in $\mu \mathrm{mol}$ per $\mathrm{g}$ of fresh weight, while lipid peroxidation was estimated as nmol of MDA per $g$ of fresh weight.

\subsubsection{Antioxidant activity assays}

The antioxidant capacity was evaluated using the 2,2-diphenyl-1picrylhydrazyl-hydrate (DPPH) and ferric reducing ability of plasma (FRAP) assays according to Wojdyło, Oszmiański, and Czemerys (2007) with some modifications (Chrysargyris et al., 2016). Antioxidant activity was estimated as Trolox equivalents (positive control) per $\mathrm{g}$ fresh weight.

\subsection{Plant and nutrient ion concentration analysis}

Minerals content (potassium (K), phosphorus (P), calcium (Ca), magnesium ( $\mathrm{Mg}$ ), sodium $(\mathrm{Na})$, iron $(\mathrm{Fe})$, manganese $(\mathrm{Mn})$, zinc $(\mathrm{Zn})$, copper ( $\mathrm{Cu}$ ), and boron (B)] was assayed by coupled plasma atomic emission spectrometry (ICP-AES; PSFO 2.0 (Leeman Labs Inc., Hudson, $\mathrm{NH})$ ) (Chrysargyris et al., 2016). N content was determined with Kjeldahl digestion method (BUCHI, Digest automat K-439 and Distillation Kjeldahl K-360).

\subsection{Essential oil extraction and gas chromatography/mass spectrometry analysis}

Spearmint aerial parts were collected after five weeks of cultivation. The fresh samples were air-dried at $42{ }^{\circ} \mathrm{C}$ in a forced-air drying oven. The dried tissues were chopped prior to analysis and essential oil was extracted with a Clevenger apparatus. Each extraction lasted for approximately $3 \mathrm{~h}$, while each treatment was replicated three times. For essential oil composition analysis, GC/MS was implemented (Shimadzu GC2010 interfaced with a Shimadzu GC/MS QP2010; Shimadzu Corporation, Kyoto, Japan). Essential oil components were identified by comparing their retention indices and mass spectra with authentic standards when available. For those compounds where no authentic standards were available, tentative identification was implemented by comparing mass spectra of components with spectral data of the built-in Mass Spectra Library NIST08 of the GC-MS system and the literature.

\subsection{Organic acids determination}

Organic acids were determined by HPLC-DAD (Shimadzu 20A series; Shimadzu Corporation, Kyoto, Japan) operating under the conditions described by Barros, Pereira, and Ferreira (2013). The results were expressed as $\mathrm{mg}$ per $100 \mathrm{~g}$ fresh weight.

\subsection{Phenolic compounds identification}

Composition of phenolic compounds of the aerial plant parts was determined by HPLC-DAD-ESI/MS (Dionex Ultimate 3000, Thermo Scientific, San Jose, CA) according to the method previously described 
by Bessada, Barreira, Barros, Ferreira, and Oliveira (2016). Calibration curves for each available phenolic standard (chlorogenic acid, apigenin6-C-glucoside, apigenin-7-O-glucoside, rosmarinic acid, quercetin-3-Oglucoside, Extrasynthèse, Genay, France) were prepared. The results were expressed as mg per $g$ of extract.

\subsection{Statistical methods}

Statistical analysis was carried out with the use of IBM SPSS v.22 (IBM Corp., Armonk, NY, USA) statistical software and results were expressed as means \pm SE ( $n=6$ for aerial parts and $n=6$ for roots). Differences between treatment means were compared at $p=0.05$ with ANOVA, followed by Duncan Multiple Range Test (DMRT) at $p=0.05$, in order to find statistically significant differences. Linear discriminant analysis (LDA) was used to determine the percentage of volatile compounds of the essential oil and the relationship among $\mathrm{P}$ concentrations.

\section{Results}

\subsection{Plant growth}

Plant growth was affected by phosphorus $(\mathrm{P})$ rates, where the addition of $50 \mathrm{mg} / \mathrm{L} \mathrm{P}$ in the nutrient solution resulted in a higher number of leaves (Fig. 1A), and more compact plants, as in the case of P70 level (Fig. 1B). Plants grown under P concentration of $50 \mathrm{mg} / \mathrm{L}$ and $70 \mathrm{mg} / \mathrm{L}$ revealed the greatest dry matter content, $16.0 \%$ and $17.3 \%$, respectively (Fig. 1C), while dry matter content of roots increased in plants grown at the highest P concentration (P70) (Fig. 1D). The biomass:root ratio of fresh weight has its maximum value of 2.10 at the $40 \mathrm{mg} / \mathrm{L}$ of $\mathrm{P}$ application, whereas no significant differences were found in plant (aerial part and roots) fresh weight, shoot number, stem thickness and root length among the tested P levels (data not shown).

\subsection{Physiological parameters}

The application of $\mathrm{P}$ in the nutrient solution had a strong effect on the studied physiological parameters (Table 1). Stomatal conductivity had its peak at $0.34 \mathrm{~cm} / \mathrm{s}$ when $40 \mathrm{mg} / \mathrm{L}$ of $\mathrm{P}$ were applied. Moreover, chlorophyll fluorescence had its lowest value $(0.76)$ at the level of P70, while chlorophyll content increased up to $1.87,0.6$ and $2.47 \mathrm{mg} / \mathrm{g}$ fresh weight for $\mathrm{Chl}$ a, Chl b and total chlorophylls, respectively, at the same level. The highest values of chlorophyll fluorescence (0.80 and 0.81$)$ were noticed at P40 and P50, respectively.

The effect of $\mathrm{P}$ application rate on antioxidant activity is presented in Fig. $1 \mathrm{E}$ and F. The highest antioxidant activity for both the tested assays was observed at P70, with significant differences between the other P levels, especially for the FRAP assay (Fig. 1F).

The effect of $\mathrm{P}$ application rate on $\mathrm{H}_{2} \mathrm{O}_{2}$ content and SOD activity is presented in Fig. 1G and $\mathrm{H}$. The increasing application of different $\mathrm{P}$ concentrations had an impact on $\mathrm{H}_{2} \mathrm{O}_{2}$ production, increasing its content up to $1.81 \mu \mathrm{mol} / \mathrm{g}$ fresh weight at P70 (Fig. 1G). SOD activity showed the opposite trend without a significant decrease at $\mathrm{P}$ levels higher than $30 \mathrm{mg} / \mathrm{L}$ being observed (Fig. $1 \mathrm{H}$ ). For the rest of the tested antioxidant enzymes (CAT and APX) and lipid peroxidation (MDA), no significant effect of P application rate was observed (data not shown).

\subsection{Leaf and root nutrient content}

Leaf and root nutrients content in relation to $\mathrm{P}$ application rates is presented in Table 2. Leaves macronutrients and micronutrients were detected in the following order regarding its content: $\mathrm{N}>\mathrm{K}>$ $\mathrm{Ca}>\mathrm{Na}>\mathrm{Mg}>\mathrm{P}$ and $\mathrm{Fe}>\mathrm{Al}>\mathrm{Mn}>\mathrm{B}>\mathrm{Zn} \approx \mathrm{Cu}$, respectively. Similarly, the following order observed for the macronutrients and micronutrients content of spearmint roots: $\mathrm{K} \geq \mathrm{N}>\mathrm{Ca}>\mathrm{Na}>$ $\mathrm{Mg} \geq \mathrm{P}$, and $\mathrm{Fe}>\mathrm{Mn} \approx \mathrm{Al}>\mathrm{B}>\mathrm{Zn}>\mathrm{Cu}$, respectively. In general, medium $\mathrm{P}$ levels (P50) resulted in mineral accumulation of $\mathrm{K}, \mathrm{Na}$,
$\mathrm{Fe}, \mathrm{Al}, \mathrm{Cu}$ and $\mathrm{B}$ in spearmint leaves comparing to low or high $\mathrm{P}$ levels, whereas $\mathrm{N}$ content declined at the P50 level. Moreover, $\mathrm{Ca}, \mathrm{Mg}$ and $\mathrm{Zn}$ remained unaffected to $\mathrm{P}$ concentrations. In roots, different $\mathrm{P}$ concentrations in the nutrient solution had a significant effect only in $\mathrm{P}$ content in terms of macronutrients, while regarding micronutrients, only $\mathrm{Al}$ and $\mathrm{Mn}$ were significantly affected. Regression analysis among $\mathrm{P}$ application rates and plant tissues mineral content revealed a significant positive correlation of $\mathrm{P}$ rate with Mn content $(p=0.0075)$ and a negative correlation with $\mathrm{K}$ content $(p=0.0063)$.

\subsection{Composition and yield of essential oil}

The increase of $\mathrm{P}$ content in the nutrient solution did not significantly affect essential oil yield of the aerial parts of spearmint (data not shown). Composition of essential oil and retention indices of volatile compounds are presented in Table 3. Essential oil analysis revealed the presence of twenty-seven individual compounds, while the detected compounds represented $98.71-99.59 \%$ of the oil in the tested $\mathrm{P}$ rates. It can be further noticed that oxygenated monoterpenes were the most abound compounds (76.03\%-80.96\%) followed by monoterpenes hydrocarbon (14.04\%-16.78\%), sesquiterpenes hydrocarbons (3.91\%-6.20\%), and sesquiterpenes $(0.04 \%-0.21 \%)$ (Table 3). The major components of the examined spearmint oil in decreasing order were carvone (68.13-74.25\%), limonene (10.07-12.08\%), 1.8-cineole (4.78-6.07\%), germacrene D (2.09-3.13\%), $\beta$-pinene (1.13-1.37\%) and $\beta$-caryophyllene $(0.80-1.29 \%)$, accounting for $91.65 \%-94.27 \%$ of total chromatographic profile. Other compounds were also identified in amounts lower than $1 \%$ of the total volatile compounds composition (Table 3).

Concerning the $\mathrm{P}$ effects on spearmint oil composition, the highest amounts of the major oil component (carvone) increased up to $74.27 \%$ and $74.25 \%$ at P70 and P50 levels, respectively. In contrast, other compounds, such as $\beta$-caryophyllene, $\beta$-myrcene and sabinene decreased at P50 level $(0.80 \%, 0.780 \%$ and $0.75 \%$, respectively). The rest of the major components were not affected significantly by P application rates (Table 3 ).

\subsection{Linear discriminant analysis}

For the effect of $\mathrm{P}$ application rate on spearmint essential oil composition, a linear discriminant analysis (LDA) was also performed. The findings of this analysis are presented in Supplementary Material Fig. S3 and showed that the first two principal axes accounted for $98.8 \%$ of total variation. The first axis $(97.8 \%$ of total variation) was mainly correlated with sabinene, $\beta$-pinene, $\beta$-myrcene, $\alpha$-pinene, $\beta$-ocimene, 1.8-cineole, camphene, cis-sabinene hydrate, and limonene. The second axis accounted for $1.0 \%$ of total variation, with 3-octanol being the only compound contributing to its definition. Projection of average values from all the essential oil compounds on the first two principal axes, suggested a significant dispersion among P levels (Supplementary Material Fig. S3). Therefore, according to the LDA, four distinct groups in relation to $\mathrm{P}$ level were observed situated at the periphery and the center of the plot. The first and second group was represented by P50 and P30 levels, respectively, situated at the negative side of axis 1 and both positive and negative side of axis 2 . The third and fourth group was represented by P40, P70 and P60, positioned at the positive side of axis 1 and both positive and negative side of axis 2 .

\subsection{Organic acids content}

Organic acids content is presented in Table 4 for four compounds, oxalic, malic, ascorbic and citric acids. Oxalic and citric acids were the richest compounds, followed by malic and ascorbic acid. Both individual compounds and total organic acids content were beneficially affected by the addition of $50 \mathrm{mg} / \mathrm{L}$ of $\mathrm{P}$ in the nutrient solution. 


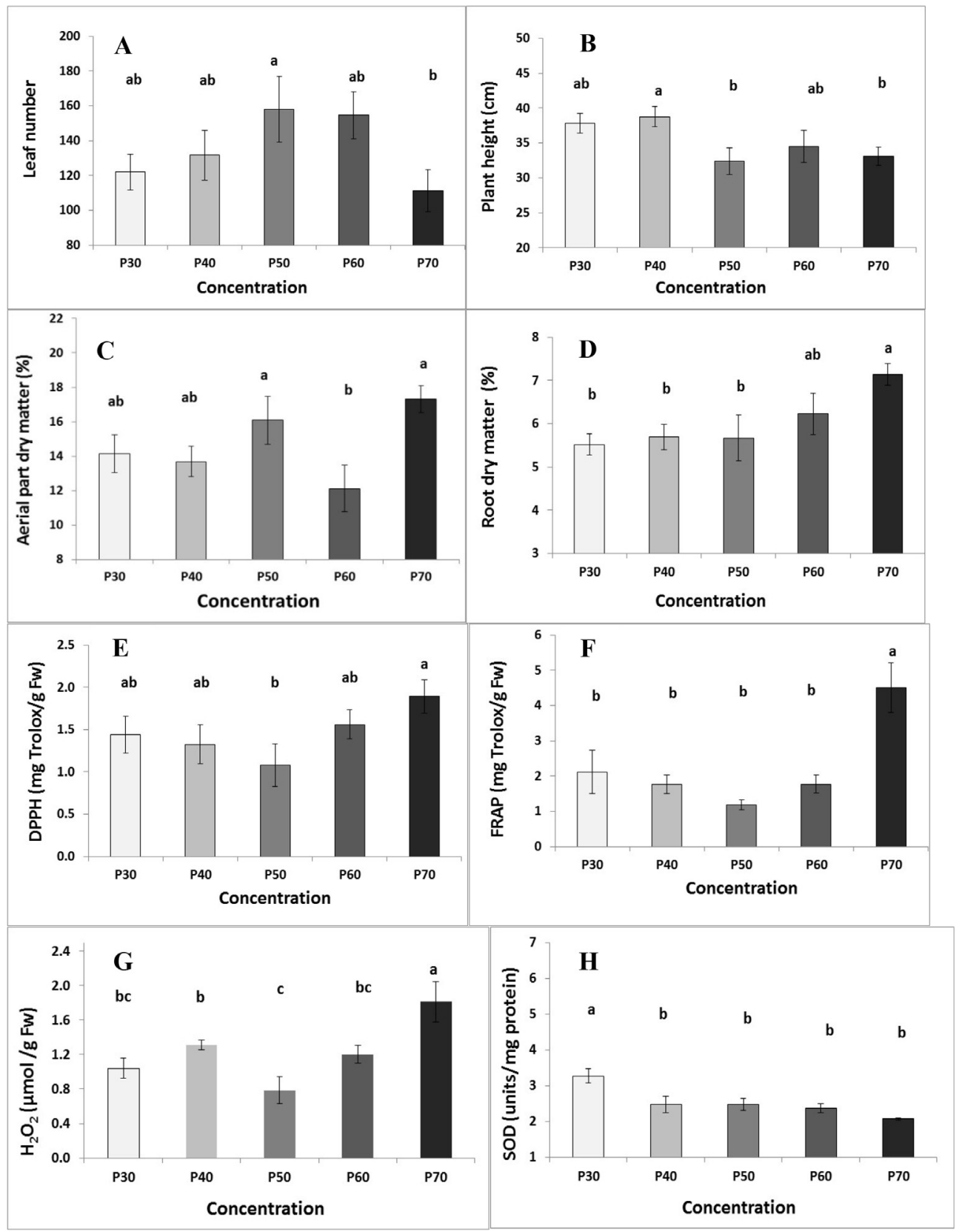

Fig. 1. Effects of phosphorus on (A) leaf number, (B) plant height, (C) aerial part dry matter content (DM; \%), (D) root dry matter content (DM; \%), (E) DPPH, (F) FRAP, (G) $\mathrm{H}_{2} \mathrm{O}_{2}$, and (H) SOD values of spearmint plants grown hydroponically in DFT. Values are expressed as means \pm SE $(n=6)$. Bars followed by the same letter are not significantly different at $p \leq 0.05$. P30, P40, P50, P60 and P70 indicate phosphorus concentration in mg/L.

\subsection{Phenolic compounds composition}

Table 5 presents information regarding chromatographic characteristics of the detected phenolic compounds in spearmint leaves.
Eleven individual phenolic compounds were detected in all the treatments, being identified as four flavonoids (3 flavones and 1 flavonol glycoside derivatives) and seven hydroxycinnamic acid derivatives (chlorogenic acid and caffeic acid derivatives). These compounds were

Table 1

Influence of different $\mathrm{P}$ levels on leaf stomatal conductivity $(\mathrm{cm} / \mathrm{s})$, leaf fluorescence $\left(\mathrm{F}_{\mathrm{v}} / \mathrm{F}_{\mathrm{m}}\right)$ and chlorophylls content (mg/g fresh weight) of spearmint plants.

\begin{tabular}{llllll}
\hline $\mathrm{P}$ & $\mathrm{P} 30$ & $\mathrm{P} 40$ & $\mathrm{P} 50$ & $\mathrm{P} 60$ & $\mathrm{P}$ \\
\hline stomatal conductivity & $0.26 \pm 0.03 \mathrm{ab}^{\mathrm{Y}}$ & $0.34 \pm 0.12 \mathrm{a}$ & $0.12 \pm 0.04 \mathrm{~b}$ & $0.24 \pm 0.06 \mathrm{ab}$ & $0.22 \pm 0.03 \mathrm{ab}$ \\
fluorescence & $0.79 \pm 0.01 \mathrm{ab}$ & $0.80 \pm 0.02 \mathrm{a}$ & $0.81 \pm 0.01 \mathrm{a}$ & $0.79 \pm 0.01 \mathrm{ab}$ & $0.76 \pm 0.01 \mathrm{~b}$ \\
chlorophyll $a$ & $1.66 \pm 0.05 \mathrm{ab}$ & $1.65 \pm 0.02 \mathrm{ab}$ & $1.65 \pm 0.05 \mathrm{ab}$ & $1.56 \pm 0.06 \mathrm{~b}$ & $1.87 \pm 0.11 \mathrm{a}$ \\
chlorophyll $b$ & $0.51 \pm 0.02 \mathrm{ab}$ & $0.53 \pm 0.01 \mathrm{ab}$ & $0.54 \pm 0.02 \mathrm{ab}$ & $0.48 \pm 0.04 \mathrm{~b}$ & $0.60 \pm 0.03 \mathrm{a}$ \\
total chlorophyll & $2.17 \pm 0.07 \mathrm{ab}$ & $2.18 \pm 0.03 \mathrm{ab}$ & $2.19 \pm 0.08 \mathrm{ab}$ & $2.05 \pm 0.15 \mathrm{~b}$ & $2.47 \pm 0.10 \mathrm{a}$
\end{tabular}

${ }^{\mathrm{Y}}$ Values are expressed as means $\pm \mathrm{SE}(n=6)$. Means in the same row followed by the same letter are not significantly different at $p \leq 0.05 ; \mathrm{P} 30, \mathrm{P} 40, \mathrm{P} 50, \mathrm{P} 60$ and P70 indicate phosphorus concentration in $\mathrm{mg} / \mathrm{L}$. 
Table 2

Effects of phosphorus (P) application rate on leaves and roots of spearmint plants.

\begin{tabular}{|c|c|c|c|c|c|}
\hline Leaves & P30 & $\mathrm{P} 40$ & P50 & $\mathrm{P} 60$ & P70 \\
\hline $\mathrm{N}(\mathrm{g} / \mathrm{kg})$ & $33.3 \pm 1.11 \mathrm{ab}^{\mathrm{Y}}$ & $34.9 \pm 1.22 \mathrm{a}$ & $30.8 \pm 0.97 b$ & $35.1 \pm 1.13 \mathrm{a}$ & $32.1 \pm 0.79 \mathrm{ab}$ \\
\hline $\mathrm{K}(\mathrm{g} / \mathrm{kg})$ & $25.8 \pm 0.37 a$ & $25.0 \pm 0.73 a$ & $24.8 \pm 1.52 \mathrm{a}$ & $24.4 \pm 1.01 \mathrm{ab}$ & $21.7 \pm 0.71 b$ \\
\hline $\mathrm{Ca}(\mathrm{g} / \mathrm{kg})$ & $8.14 \pm 0.43 a$ & $8.43 \pm 0.51 \mathrm{a}$ & $8.62 \pm 0.99 a$ & $8.41 \pm 0.35 a$ & $7.87 \pm 0.31 \mathrm{a}$ \\
\hline$P(g / k g)$ & $2.06 \pm 0.04 a$ & $2.25 \pm 0.10 \mathrm{a}$ & $2.38 \pm 0.19 a$ & $2.26 \pm 0.06 \mathrm{a}$ & $2.20 \pm 0.13 a$ \\
\hline $\mathrm{Mg}(\mathrm{g} / \mathrm{kg})$ & $2.50 \pm 0.10 \mathrm{a}$ & $2.58 \pm 0.11 \mathrm{a}$ & $2.45 \pm 0.12 \mathrm{a}$ & $2.48 \pm 0.06 a$ & $2.48 \pm 0.08 \mathrm{a}$ \\
\hline $\mathrm{Na}(\mathrm{g} / \mathrm{kg})$ & $2.41 \pm 0.27 b$ & $2.59 \pm 0.21 b$ & $3.35 \pm 0.10 \mathrm{a}$ & $2.24 \pm 0.07 b$ & $2.76 \pm 0.22 b$ \\
\hline $\mathrm{Al}(\mathrm{mg} / \mathrm{kg})$ & $37.80 \pm 2.15 b$ & $32.3 \pm 2.64 b$ & $72.6 \pm 7.50 \mathrm{a}$ & $38.7 \pm 2.77 b$ & $37.9 \pm 4.15 b$ \\
\hline $\mathrm{Fe}(\mathrm{mg} / \mathrm{kg})$ & $108 \pm 3.73 b$ & $119 \pm 5.39 b$ & $137 \pm 7.65 \mathrm{a}$ & $107 \pm 4.52 b$ & $104 \pm 4.19 b$ \\
\hline $\mathrm{Mn}(\mathrm{mg} / \mathrm{kg})$ & $37.7 \pm 4.19 c$ & $51.4 \pm 6.71 \mathrm{ab}$ & $44.5 \pm 3.22 b c$ & $60.7 \pm 3.59 a$ & $53.9 \pm 4.31 \mathrm{ab}$ \\
\hline $\mathrm{Zn}(\mathrm{mg} / \mathrm{kg})$ & $14.4 \pm 1.08 \mathrm{a}$ & $14.0 \pm 2.47 \mathrm{a}$ & $15.8 \pm 1.48 \mathrm{a}$ & $13.3 \pm 1.65 a$ & $11.9 \pm 0.90 \mathrm{a}$ \\
\hline $\mathrm{Cu}(\mathrm{mg} / \mathrm{kg})$ & $8.67 \pm 0.36 b c$ & $9.55 \pm 0.65 b$ & $13.2 \pm 0.81 \mathrm{a}$ & $9.27 \pm 0.53 b$ & $7.32 \pm 0.52 c$ \\
\hline $\mathrm{B}(\mathrm{mg} / \mathrm{kg})$ & $54.7 \pm 5.69 a$ & $38.8 \pm 2.30 \mathrm{~b}$ & $55.2 \pm 1.34 \mathrm{a}$ & $39.9 \pm 1.06 \mathrm{~b}$ & $45.4 \pm 2.38 b$ \\
\hline Roots & P30 & $\mathrm{P} 40$ & P50 & P60 & P70 \\
\hline $\mathrm{N}(\mathrm{g} / \mathrm{kg})$ & $29.4 \pm 0.69 \mathrm{a}^{\mathrm{Y}}$ & $27.9 \pm 1.59 \mathrm{a}$ & $26.6 \pm 0.35 a$ & $27.1 \pm 0.44 a$ & $27.5 \pm 1.16 \mathrm{a}$ \\
\hline $\mathrm{K}(\mathrm{g} / \mathrm{kg})$ & $33.7 \pm 1.20 \mathrm{a}$ & $29.3 \pm 3.32 \mathrm{a}$ & $32.3 \pm 0.11 \mathrm{a}$ & $29.6 \pm 1.25 \mathrm{a}$ & $31.0 \pm 1.53 \mathrm{a}$ \\
\hline $\mathrm{Ca}(\mathrm{g} / \mathrm{kg})$ & $12.6 \pm 2.28 \mathrm{a}$ & $13.0 \pm 2.30 \mathrm{a}$ & $14.6 \pm 1.13 \mathrm{a}$ & $12.8 \pm 0.55 a$ & $15.8 \pm 0.86 \mathrm{a}$ \\
\hline$P(g / k g)$ & $2.58 \pm 0.20 c$ & $3.07 \pm 0.05 b c$ & $3.91 \pm 0.27 \mathrm{ab}$ & $3.40 \pm 0.15 b c$ & $5.05 \pm 0.52 \mathrm{a}$ \\
\hline $\mathrm{Mg}(\mathrm{g} / \mathrm{kg})$ & $2.57 \pm 0.11 \mathrm{a}$ & $2.57 \pm 0.11 \mathrm{a}$ & $2.71 \pm 0.09 a$ & $2.67 \pm 0.11 \mathrm{a}$ & $2.57 \pm 0.16 a$ \\
\hline $\mathrm{Na}(\mathrm{g} / \mathrm{kg})$ & $3.45 \pm 0.34 a$ & $3.46 \pm 0.27 a$ & $3.68 \pm 0.24 a$ & $3.03 \pm 0.12 a$ & $3.49 \pm 0.28 \mathrm{a}$ \\
\hline $\mathrm{Al}(\mathrm{mg} / \mathrm{kg})$ & $290 \pm 34.6 a$ & $150 \pm 14.2 b$ & $346 \pm 8.76 a$ & $168 \pm 13.9 b$ & $156 \pm 12.7 b$ \\
\hline $\mathrm{Fe}(\mathrm{mg} / \mathrm{kg})$ & $849 \pm 83.9 a$ & $948 \pm 176 a$ & $1005 \pm 113 a$ & $806 \pm 53.5 a$ & $954 \pm 111 a$ \\
\hline $\mathrm{Zn}(\mathrm{mg} / \mathrm{kg})$ & $43.8 \pm 3.06 \mathrm{a}$ & $57.8 \pm 5.48 \mathrm{a}$ & $53.3 \pm 8.97 a$ & $48.0 \pm 2.88 \mathrm{a}$ & $52.9 \pm 8.45 a$ \\
\hline $\mathrm{Cu}(\mathrm{mg} / \mathrm{kg})$ & $126 \pm 14.6 a$ & $154 \pm 17.1 \mathrm{a}$ & $164 \pm 5.54 a$ & $144 \pm 5.69 a$ & $143 \pm 14.2 \mathrm{a}$ \\
\hline $\mathrm{B}(\mathrm{mg} / \mathrm{kg})$ & $35.5 \pm 3.48 \mathrm{a}$ & $30.2 \pm 1.98 \mathrm{a}$ & $34.5 \pm 2.89 a$ & $29.7 \pm .12 \mathrm{a}$ & $37.0 \pm 1.18 \mathrm{a}$ \\
\hline
\end{tabular}

${ }^{\mathrm{Y}}$ Values are expressed as means $\pm \mathrm{SE}$ ( $n=6$ in the case of leaves and $n=3$ in the case of roots). Means in the same row followed by the same letter are not significantly different at $p \leq 0.05$; P30, P40, P50, P60 and P70 indicate phosphorus concentration in mg/L.

Table 3

Composition (\%) of essential oil of hydroponically grown spearmint plants in relation to phosphorus application rates.

\begin{tabular}{|c|c|c|c|c|c|c|}
\hline \multirow[t]{2}{*}{ compound } & \multirow[t]{2}{*}{ RI } & \multicolumn{5}{|l|}{$\mathrm{P}$ concentrations } \\
\hline & & P30 & $\mathrm{P} 40$ & P50 & P60 & P70 \\
\hline$\alpha$-pinene & 933 & $0.93 \pm 0.03 \mathrm{a}^{\mathrm{Y}}$ & $0.91 \pm 0.00 \mathrm{a}$ & $0.81 \pm 0.07 a$ & $0.88 \pm 0.03 a$ & $0.78 \pm 0.03 \mathrm{a}$ \\
\hline camphene & 948 & $0.05 \pm 0.00 \mathrm{a}$ & $0.05 \pm 0.00 \mathrm{a}$ & $0.03 \pm 0.00 \mathrm{~b}$ & $0.06 \pm 0.00 \mathrm{a}$ & $0.06 \pm 0.00 \mathrm{a}$ \\
\hline sabinene & 973 & $0.88 \pm 0.03 a$ & $0.79 \pm 0.00 \mathrm{ab}$ & $0.75 \pm 0.05 b$ & $0.79 \pm 0.02 \mathrm{ab}$ & $0.79 \pm 0.01 \mathrm{ab}$ \\
\hline$\beta$-pinene & 977 & $1.30 \pm 0.06 \mathrm{a}$ & $1.25 \pm 0.02 \mathrm{a}$ & $1.17 \pm 0.07 a$ & $1.25 \pm 0.04 \mathrm{a}$ & $1.14 \pm 0.08 \mathrm{a}$ \\
\hline$\beta$-myrcene & 991 & $0.92 \pm 0.07 a$ & $0.90 \pm 0.03 a$ & $0.80 \pm 0.06 \mathrm{~b}$ & $0.90 \pm 0.01 \mathrm{a}$ & $0.90 \pm 0.03 a$ \\
\hline 3-octanol & 995 & $0.13 \pm 0.00 \mathrm{bc}$ & $0.14 \pm 0.00 \mathrm{~b}$ & $0.12 \pm 0.01 c$ & $0.13 \pm 0.00 b c$ & $0.16 \pm 0.00 \mathrm{a}$ \\
\hline limonene & 1028 & $10.60 \pm 0.17 \mathrm{a}$ & $10.96 \pm 0.35 \mathrm{a}$ & $10.42 \pm 0.28 \mathrm{a}$ & $10.82 \pm 0.03 a$ & $10.07 \pm 0.38 \mathrm{a}$ \\
\hline 1.8-cineole & 1031 & $5.27 \pm 0.21 \mathrm{a}$ & $5.52 \pm 0.27 \mathrm{a}$ & $5.54 \pm 0.04 a$ & $5.58 \pm 0.15 \mathrm{a}$ & $4.78 \pm 0.45 a$ \\
\hline$\beta$-ocimene & 1036 & $0.35 \pm 0.01 \mathrm{a}$ & $0.32 \pm 0.01 \mathrm{a}$ & $0.25 \pm 0.02 b$ & $0.35 \pm 0.01 \mathrm{a}$ & $0.30 \pm 0.01 \mathrm{a}$ \\
\hline cis-sabinene hydrate & 1067 & $0.37 \pm 0.04 \mathrm{~b}$ & $0.44 \pm 0.01 \mathrm{ab}$ & $0.36 \pm 0.00 \mathrm{~b}$ & $0.44 \pm 0.00 \mathrm{ab}$ & $0.49 \pm 0.06 \mathrm{a}$ \\
\hline borneol & 1166 & $0.24 \pm 0.01 \mathrm{a}$ & $0.25 \pm 0.00 \mathrm{a}$ & $0.23 \pm 0.01 \mathrm{a}$ & $0.31 \pm 0.00 \mathrm{~b}$ & $0.32 \pm 0.02 b$ \\
\hline terpinen-4-ol & 1178 & $0.08 \pm 0.01 \mathrm{ab}$ & $0.04 \pm 0.00 c$ & $0.05 \pm 0.00 b c$ & $0.09 \pm 0.00 \mathrm{a}$ & $0.08 \pm 0.00 \mathrm{ab}$ \\
\hline$\alpha$-terpineol & 1191 & $0.18 \pm 0.01 \mathrm{a}$ & $0.18 \pm 0.00 \mathrm{a}$ & $0.16 \pm 0.01 \mathrm{a}$ & $0.21 \pm 0.00 \mathrm{a}$ & $0.15 \pm 0.03 a$ \\
\hline cis-dihydrocarvone & 1198 & $0.15 \pm 0.02 \mathrm{a}$ & $0.13 \pm 0.01 \mathrm{ab}$ & $0.08 \pm 0.02 b$ & $0.15 \pm 0.00 \mathrm{a}$ & $0.14 \pm 0.01 \mathrm{a}$ \\
\hline trans-carveol & 1219 & $0.05 \pm 0.01 b c$ & $0.04 \pm 0.00 c$ & $0.01 \pm 0.01 \mathrm{c}$ & $0.13 \pm 0.00 \mathrm{a}$ & $0.09 \pm 0.02 \mathrm{ab}$ \\
\hline cis-carveol & 1231 & $0.12 \pm 0.01 \mathrm{a}$ & $0.05 \pm 0.00 \mathrm{~b}$ & $0.03 \pm 0.02 b$ & $0.16 \pm 0.00 \mathrm{a}$ & $0.10 \pm 0.03 a$ \\
\hline pulegone & 1240 & $0.30 \pm 0.01 \mathrm{a}$ & $0.32 \pm 0.02 \mathrm{a}$ & $0.20 \pm 0.01 b$ & $0.33 \pm 0.01 \mathrm{a}$ & $0.30 \pm 0.01 \mathrm{a}$ \\
\hline carvone & 1244 & $72.19 \pm 0.53 \mathrm{ab}$ & $71.28 \pm 0.59 b$ & $74.25 \pm 0.44 a$ & $70.29 \pm 0.51 b$ & $74.27 \pm 0.65 a$ \\
\hline$\beta$-bourbonene & 1386 & $0.20 \pm 0.01 \mathrm{ab}$ & $0.22 \pm 0.02 \mathrm{a}$ & $0.16 \pm 0.01 b$ & $0.22 \pm 0.00 \mathrm{a}$ & $0.19 \pm 0.01 \mathrm{ab}$ \\
\hline$\beta$-elemene & 1393 & $0.31 \pm 0.02 \mathrm{a}$ & $0.31 \pm 0.03 a$ & $0.17 \pm 0.01 b$ & $0.34 \pm 0.01 \mathrm{a}$ & $0.20 \pm 0.04 a$ \\
\hline$\beta$-caryophyllene & 1425 & $1.05 \pm 0.03 a$ & $1.13 \pm 0.10 \mathrm{a}$ & $0.80 \pm 0.00 \mathrm{~b}$ & $1.16 \pm 0.03 a$ & $1.01 \pm 0.09 \mathrm{a}$ \\
\hline germacrene D & 1497 & $2.28 \pm 0.04 a$ & $2.50 \pm 0.20 \mathrm{a}$ & $2.09 \pm 0.05 a$ & $2.55 \pm 0.10 \mathrm{a}$ & $2.09 \pm 0.24 \mathrm{a}$ \\
\hline bicyclogermacrene & 1512 & $0.75 \pm 0.03 \mathrm{ab}$ & $0.87 \pm 0.11 \mathrm{a}$ & $0.60 \pm 0.02 b$ & $0.91 \pm 0.02 \mathrm{a}$ & $0.87 \pm 0.10 \mathrm{a}$ \\
\hline germacrene A & 1519 & $0.11 \pm 0.00 \mathrm{a}$ & $0.14 \pm 0.03 a$ & $0.02 \pm 0.01 b$ & $0.17 \pm 0.01 \mathrm{a}$ & $0.11 \pm 0.03 a$ \\
\hline trans-calamene & 1534 & $0.00 \pm 0.00 \mathrm{~b}$ & $0.00 \pm 0.00 \mathrm{~b}$ & $0.05 \pm 0.00 \mathrm{a}$ & $0.06 \pm 0.01 \mathrm{a}$ & $0.04 \pm 0.00 \mathrm{a}$ \\
\hline 1,10-di-epicubenol & 1617 & $0.07 \pm 0.01 \mathrm{ab}$ & $0.07 \pm 0.01 \mathrm{ab}$ & $0.02 \pm 0.01 b$ & $0.09 \pm 0.00 \mathrm{a}$ & $0.10 \pm 0.03 a$ \\
\hline$\alpha$-cadinol & 1657 & $0.05 \pm 0.01 \mathrm{a}$ & $0.06 \pm 0.01 \mathrm{a}$ & $0.02 \pm 0.01 \mathrm{a}$ & $0.07 \pm 0.00 \mathrm{a}$ & $0.06 \pm 0.02 \mathrm{a}$ \\
\hline \multicolumn{2}{|c|}{ monoterpenes hydrocarbons } & $15.05 \pm 0.12 \mathrm{a}$ & $15.21 \pm 0.32 \mathrm{a}$ & $14.24 \pm 0.55 \mathrm{a}$ & $15.05 \pm 0.08 \mathrm{a}$ & $14.04 \pm 0.07 \mathrm{a}$ \\
\hline \multicolumn{2}{|c|}{ sesquiterpenes hydrocarbons } & $4.72 \pm 0.15 \mathrm{ab}$ & $5.18 \pm 0.51 \mathrm{a}$ & $3.91 \pm 0.01 b$ & $5.42 \pm 0.21 \mathrm{a}$ & $4.51 \pm 0.55 a$ \\
\hline \multicolumn{2}{|c|}{ oxygenated monoterpenes } & $78.97 \pm 0.35 b$ & $78.27 \pm 0.97 b$ & $80.96 \pm 0.56 a$ & $78.00 \pm 0.37 b$ & $80.72 \pm 0.67 \mathrm{a}$ \\
\hline \multicolumn{2}{|c|}{ oxygenated sesquiterpenes } & $0.13 \pm 0.02 \mathrm{ab}$ & $0.14 \pm 0.03 \mathrm{ab}$ & $0.04 \pm 0.02 b$ & $0.17 \pm 0.00 \mathrm{a}$ & $0.16 \pm 0.05 a$ \\
\hline \multicolumn{2}{|c|}{ others } & $0.13 \pm 0.00 \mathrm{bc}$ & $0.14 \pm 0.00 \mathrm{~b}$ & $0.12 \pm 0.01 c$ & $0.13 \pm 0.00 b c$ & $0.16 \pm 0.00 \mathrm{a}$ \\
\hline \multicolumn{2}{|l|}{ total } & $99.02 \pm 0.08 \mathrm{a}$ & $98.95 \pm 0.17 \mathrm{a}$ & $99.28 \pm 0.01 \mathrm{a}$ & $98.87 \pm 0.06 \mathrm{a}$ & $99.59 \pm 0.10 \mathrm{a}$ \\
\hline
\end{tabular}

$\mathrm{Y}$ Values are expressed as means $\pm \mathrm{SE}(n=3)$. Means in rows followed by the same letter are not significantly different at $p \leq 0.05$; P30, P40, P50, P60 and P70 indicate phosphorus concentration in $\mathrm{mg} / \mathrm{L}$. 
Table 4

Composition in organic acids of hydroponically grown spearmint plants in relation to phosphorus application rates (g/100 $\mathrm{g}$ dw) (mean \pm SD).

\begin{tabular}{|c|c|c|c|c|c|}
\hline & oxalic $\operatorname{acid}^{Y}$ & malic acid & ascorbic acid & citric acid & total organic acids \\
\hline P30 & $4.85 \pm 0.05 b$ & $1.06 \pm 0.03 c$ & $0.030 \pm 0.001 \mathrm{~d}$ & $4.2 \pm 0.1 c$ & $10.1 \pm 0.2 \mathrm{c}$ \\
\hline P40 & $4.71 \pm 0.01 d$ & $1.17 \pm 0.03 b$ & $0.030 \pm 0.001 d$ & $4.37 \pm 0.04 b$ & $10.29 \pm 0.01 b$ \\
\hline P50 & $5.01 \pm 0.01 \mathrm{a}$ & $1.08 \pm 0.02 c$ & $0.300 \pm 0.002 a$ & $4.90 \pm 0.06 a$ & $11.29 \pm 0.06 \mathrm{a}$ \\
\hline P60 & $4.78 \pm 0.01 c$ & $1.29 \pm 0.03 a$ & $0.200 \pm 0.002 b$ & $4.90 \pm 0.04 a$ & $11.17 \pm 0.01 \mathrm{a}$ \\
\hline P70 & $4.32 \pm 0.02 \mathrm{e}$ & $0.84 \pm 0.02 \mathrm{~d}$ & $0.150 \pm 0.001 c$ & $3.35 \pm 0.09 \mathrm{~d}$ & $8.7 \pm 0.1 d$ \\
\hline
\end{tabular}

$\mathrm{Y}$ Values are expressed as means $\pm \mathrm{SE}(n=3)$. Means in the same column followed by the same letter are not significantly different at $p \leq 0.05$; P30, P40, P50, $\mathrm{P} 60$ and P70 indicate phosphorus concentration in $\mathrm{mg} / \mathrm{L}$.

detected in all the samples with the exception of samples treated with $50 \mathrm{mg} / \mathrm{L}$ and $70 \mathrm{mg} / \mathrm{L}$ of $\mathrm{P}$.

Rosmarinic acid was the richest phenolic compound followed by isomer 1 of lithospheric acid A (only in the case of $50 \mathrm{mg} / \mathrm{L} \mathrm{P}$ ), luteolin7-O-glucuronide and isomer 1 of salvianolic acid A (Table 5). Phenolic acids were the richest class of phenolic compound in all cases, while the application of $50 \mathrm{mg} / \mathrm{L}$ resulted in higher total phenolic compounds content, mostly because of the increased amounts of isomer 1 of lithospermic acid A and isomer 2 of salvianolic acid A. Regarding individual compounds, rosmarinic acid which is considered the most significant phenolic compound in spearmint leaves increased significantly when $70 \mathrm{mg} / \mathrm{L} \mathrm{P}$ were applied.

\section{Discussion}

Many spices and herbs are commonly used as flavoring agents in various dishes, while they are also considered as important natural sources of antioxidants. Moreover, a lot of reports have studied their effectiveness as alternative remedies for treatment of various infectious diseases. Apart from its nutritional value, spearmint (M. spicata) is a common ingredient in the cosmetics, drink, paint, and tobacco industries, and is also considered a rich source of antioxidant compounds (Nozad et al., 2016).

In the present study the effect of P levels on hydroponically grown spearmint plants in a DFT system was examined. For this purpose, plant growth, physiological parameters, such as chlorophyll determination, activities of antioxidant enzymes, damage index, antioxidant activity, mineral composition, yield and composition of essential oil, and organic acids and phenolic compounds content were measured.

\subsection{Plant growth}

The results showed that increasing P content in nutrient solution did not affect important growth parameters, such as aerial part fresh weight and shoot number, which define biomass yield. However, a beneficial effect of P on dry weight was observed, especially for the P50 and P70 levels, as well a negative effect on plant height at the same levels, which resulted in more compact plants. Considering that spearmint is usually used as dry herb, the application of medium to high P levels could increase total yield through higher plant density and dry matter content, compared to lower P levels. The benefits of dry matter yield associated with increased P levels up to $70 \mathrm{mg} / \mathrm{L}$ in the current study are in accordance with previous works on garden sage Salvia officinalis L., where biomass and essential oil content increased with increasing $\mathrm{P}$ fertilizer rates (Nell et al., 2009).

\subsection{Physiological parameters}

The rate of photosynthetic leaf capacity is associated with the chloroplast ultrastructure, the chlorophyll content and the stomatal conductance (Zhao, Oosterhuis, \& Bednarz, 2001). Chlorophyll content was higher in P70, a finding that according to Reich, Oleksyn, and Wright (2009) could be explained by the fact that photosynthetic capacity is positively correlated with $\mathrm{P}$ content, while P-deficiency affects ribulose-1,5-bisphosphate regeneration and consequently the function of photosynthetic mechanism.

There is a great interest in natural matrices containing phenolic constituents because they chelate iron and copper ions, inhibit lipid peroxidation, scavenge free radicals, and could improve the nutritional value of food products, minimizing potential problems caused by high consumption of synthetic compounds (Scherer et al., 2013). Consumers and market/industry prefer natural and safer antioxidants as food preservatives, with high antioxidant capacity (Gülçin, Elias, Gepdiremen, \& Boyer, 2006). In the present work, increasing phosphorus concentration (up to $70 \mathrm{mg} / \mathrm{L} \mathrm{P}$ ) resulted in enhanced antioxidative capacity (DPPH and FRAP) of spearmint plants.

The increased ROS scavenging capacity by plants is directly correlated with the increasing antioxidant enzymes content (SOD, APX, GR, CAT, POD) activities and/or contents (Foyer \& Noctor, 2011). Production of $\mathrm{H}_{2} \mathrm{O}_{2}$ can be eliminated through the ascorbate-glutathione cycle AsA-GSH, since according to Pasternak, Rudas, Potters, and Jansen (2005) APX and SOD are the key enzymes in this cycle. In the case of P levels, neither MDA production nor APX and CAT activities were changed. However, the increased hydrogen peroxide content in leaves at P70 resulted in high non-enzymatic antioxidant activity.

\subsection{Nutrient uptake}

The application of different levels of $\mathrm{P}$ in nutrient solution affected the content of macronutrients and micronutrients in leaves and roots. Increasing $\mathrm{P}$ concentration affected mainly micronutrients and to a lesser extent macronutrients (mainly nitrogen, calcium, sodium and potassium) in both leaves and roots. The most common interaction of $P$ is with zinc. It is well reported that excessive rates of $\mathrm{P}$ fertilizers can result in other nutrient deficiencies and vice versa. Therefore, $\mathrm{P}$ and other nutrients content has to be adjusted based on their individual concentration levels and not according to their related ratios (Sanchez, 2016). For the concentrations of $P$ tested in this study, no excess or deficit of any micronutrient was indicated, as $70 \mathrm{mg} / \mathrm{L} \mathrm{P}$ is not considered a toxic level.

\subsection{Essential oil yield and constituents}

Reports in the literature regarding EO composition of Mentha genus refer to soil production systems, while only a few studies have been conducted with plants grown in soilless conditions and controlled environments regarding the chemical composition of essential oil (Chrysargyris et al., 2017). The EO yield and composition in aromatic plants may increase with the appropriate fertigation schedule (Ramezani et al., 2009). Essential oil yield did not change by increasing $\mathrm{P}$ concentration, in accordance with previous studies on savory ( $\mathrm{Sa}$ tureja hortensis L; Alizadeh et al. (2010). In contrast, Yeşil and Kara (2016) reported a significant effect of nitrogen and phosphorus application rates on EO composition of spearmint and 1.8-cineole, 4-terpineol, pulegone, piperitone, and carvone contents in particular. These differences could be due to the interaction between nitrogen and phosphorus doses and spearmint genotypes observed in that study, compared to our study where only the effect of phosphorus dose was 
examined, as well as to different cultivation systems implemented (soil cultivation $v s$ hydroponic system). Alizadeh et al. (2010) indicated that the application of chemical fertilizers may increase the yield of essential oil and the main components content in medicinal plants.

In this study, spearmint EO was characterized by the abundance of carvone, while Scherer et al. (2013) and Chauhan et al. (2009) have also detected high levels of carvone (up to $67.1 \%$ and $76.6 \%$, respectively). Considering that quality requirements for medicinal uses of spearmint oil indicate a minimum of $51 \%$ carvone with limonene being also present (De Carvalho \& Da Fonseca, 2006), the application of P in nutrient solution helps to maintain quality, while at the same time plant density and dry matter increase may increase total oil yield per cultivated area.

It has been also noted that $M$. spicata contains high amounts of carvone (60-70\%) and limonene content within the range of 8-15\% (De Carvalho \& Da Fonseca, 2006), in agreement with leaf oil constituents of the present study, indicating also the carvone-chemotype of the selected spearmint. Oil with high carvone content receives high price in the market due to its use in several industrial applications (De Carvalho \& Da Fonseca, 2006). Therefore there is great interest in increasing carvone content of $M$. spicata through breeding and modern biotechnological tools. According to Chauhan et al. (2009), a few improved strains with high carvone content $(>60 \%$ ) are available on the market, such as MSS-1, MSS-5 and Punjab Spearmint-1. Therefore, apart from fertilization regimes, genotype selection is pivotal for the production of end-products with high quality and high added value.

1,8-Cineole or eucalyptol, is commonly used in cosmetics, fragrances and flavorings due to its characteristic aroma and taste. It also has insecticidal and insect repellent properties (Sfara, Zerba, \& Alzogaray, 2009). The capacity for oxidation inhibition of EO compounds, such as monoterpenes, oxygenated sesquiterpenes, and phenylpropanoids, has been reported (Ruberto, Baratta, Dean, \& Dorman, 2000). Similar biological activity has also been observed in the present study, where oil is rich in monoterpenes such as limonene, 1,8-cineole, and carvone.

Monoterpenes were the most abundant class compared to sesquiterpenes in the EO of the present study. This indicates that 1-deoxy-Dxylulose-5-phosphate (DXP) pathway activation is the prevalent pathway, compared with the more common mevalonic acid (MVA) pathway, which produces biosynthetic precursors of sesquiterpenes (detected in low amounts in the present study) and triterpenes.

\subsection{Organic acids content}

The main identified organic acids were oxalic and citric acid, while the ratio of oxalic acid $(\mathrm{g} / \mathrm{kg}) / \mathrm{Ca}(\mathrm{g} / \mathrm{kg}$ ) ranged between 0.16 (at P60) and 0.21 (at P50). In any case, the detected ratios were significantly lower than 2.5, which is the suggested threshold for food products to be considered as decalcifiers and poor sources of Ca (Guil, Torija, Giménez, Rodríguez-García, \& Himénez, 1996). According to Cirlini et al. (2016), organic acids such as citric and malic acid were detected in dried spearmint extracts, without however carrying out quantification of the detected compounds. Considering the role of organic acids as antioxidants (Pereira, Barros, Carvalho, \& Ferreira, 2013), this difference could be due to different growing conditions and post-harvest processing. This response has been already noted for the antioxidant activity of spearmint plants grown under open field and protected conditions (Al-Tawaha, Al-Karaki, \& Massadeh, 2013).

\subsection{Phenolic compounds composition}

The phenolic compounds detected in our study have been previously reported in Mentha species, such as infusions of Mentha spicata (Rita et al., 2016) and Mentha $\times$ piperita L. (E. Pereira et al., 2016) from Portugal. Compounds 3 (luteolin-7-O-rutinoside), 4 (luteolin-7-O-glucuronide), and 6 (rosmarinic acid) were positively identified based on 
the comparison of chromatographic and MS characteristics with commercial standards. Compounds 1, 5, 8, 9, 10 and 11 corresponded to phenolic acid derivatives. Compound $1\left([\mathrm{M}-\mathrm{H}]^{-}\right.$at $m / z$ 353) was identified as 4-O-caffeoylquinic acid taking into account the fragmentation pattern presented by Clifford, Knight, and Kuhnert (2005). Peak $5\left([\mathrm{M}-\mathrm{H}]^{-}\right.$at $\left.m / z 719\right)$ presented a base peak at $m / z 359$ which corresponded to a rosmarinic acid dimer (caffeic acid tetramer), being identified as sagerinic acid (Dias, Barros, Sousa, \& Ferreira, 2012). Peaks 8 and $9\left([\mathrm{M}-\mathrm{H}]^{-}\right.$at $\left.m / z 537\right)$ presented a fragmentation pattern comparable to lithospermic acid A (caffeic acid trimer), therefore being identified as isomers 1 and 2 (Dias et al., 2012). Compounds 10 and 11 $\left([\mathrm{M}-\mathrm{H}]^{-}\right.$at $m / z$ 493) presented a fragmentation pattern and UV spectrum similar to salvianolic acid A, being identified as isomers 1 and 2 , respectively (Dias et al., 2012). The rest of the compounds were identified as flavonoid derivatives. Peak $2\left([\mathrm{M}-\mathrm{H}]^{-}\right.$at $\left.\mathrm{m} / \mathrm{z} 593\right)$ was tentatively identified as apigenin 6,8-di-C-glucoside (vicenin-2), taking into account the findings of Ferreres et al. (2003). Compound 7 $\left([\mathrm{M}-\mathrm{H}]^{-}\right.$at $m / z$ 461) presented a UV spectrum characteristic of kaempferol $\left(\lambda_{\max }\right.$ at $\left.269,343 \mathrm{~nm}\right)$, releasing a fragment at $m / z 285$ $\left([\mathrm{M}-176]^{-}\right.$, loss of a glucuronyl moiety), being identified as kaempferol-O-glucuronide.

Rosmarinic acid was the most abundant compound, especially at high P levels (P70). Similar findings have been reported by Kivilompolo and Hyötyläinen (2007), who also identified rosmarinic acid as the most abundant phenolic compound, a common finding for aromatic herbs of the Lamiaceae family. In addition, Narasimhamoorthy, Zhao, Liu, Yang, and Greaves (2015) suggested that genotype is a determining factor for rosmarinic acid content of spearmint, which may significantly vary among different genotypes with values ranging from 7.1 to $118 \mathrm{mg} / \mathrm{g}$ DW. The results of our study showed that high P application rates may significantly increase rosmarinic acid and total phenols content, even in the case of genotypes that originally contain high amounts of polyphenols. Therefore, apart from the selection of the proper genotype, cultivation practices and growing conditions are also crucial in order to produce a high quality end-product with increased added value.

\section{Conclusion}

In summary, our findings suggest that spearmint growth and development and essential oil production may be influenced by phosphorus content in the nutrient solution. Phosphorus is considered a nutrient important for plant growth and development, interfering with secondary metabolism of plants. The increased P levels (up to $70 \mathrm{mg} / \mathrm{L}$ ) resulted in an increase of dry matter content without significant differences being observed on a fresh weight basis. The application of $70 \mathrm{mg} / \mathrm{L}$ of $\mathrm{P}$ resulted in a significant increase of carvone and rosmarinic acid content, whereas oxalic acid decreased at the same level. Therefore, this rate could be suggested for spearmint hydroponic cultivation, especially for plants grown for essential oil production and pharmaceutical industry purposes. Moreover, antioxidant activity and phenolic compounds content were beneficially affected at the highest level of $\mathrm{P}$ application rates, highlighting the importance of controlling the nutrient solution composition in hydroponically grown spearmint plants. Therefore, nutrient solution management could be considered as a way to produce end-products of high added value and high quality. Moreover, it could be also used to produce tailor-made products regarding bioactive compounds content and essential oil composition.

\section{Acknowledgements}

The authors are grateful to the project AgroLabs that has been developed under the Programme Interreg V-B Balkan - Mediterranean 2014-2020, co-funded by the European Union and National Funds of the participating countries. Also, the authors are grateful to the Foundation for Science and Technology (, Portugal) and FEDER under
Programme PT2020 for financial support to CIMO (UID/AGR/00690/ 2013), A. Fernandes (SFRH/BPD/114753/2016) and L. Barros contract; also to FEDER-Interreg España-Portugal programme for financial support through the project 0377_Iberphenol_6_E.

\section{Appendix A. Supplementary data}

Supplementary data to this article can be found online at https:// doi.org/10.1016/j.foodchem.2018.10.020.

\section{References}

Alizadeh, A., Khoshkhui, M., Javidnia, K., Firuzi, O., Tafazoli, E., \& Khalighi, A. (2010). Effects of fertilizer on yield, essential oil composition, total phenolic content and antioxidant activity in Satureja hortensis L. (Lamiaceae) cultivated in Iran. Journal of Medicinal Plants Research, 4(1), 33-40. https://doi.org/10.5897/JMPR09.361.

Al-Tawaha, A., Al-Karaki, G., \& Massadeh, A. (2013). Comparative response of essential oil composition, antioxidant activity and phenolic contents spearmint (Mentha spicata L.) under protected soilless vs. open field. Advances in Environmental Biology, 7(5), 902-910.

Barros, L., Pereira, C., \& Ferreira, I. C. F. R. (2013). Optimized analysis of organic acids in edible mushrooms from Portugal by ultra fast liquid chromatography and photodiode array detection. Food Analytical Methods, 6(1), 309-316. https://doi.org/10.1007/ s12161-012-9443-1.

Bessada, S. M. F., Barreira, J. C. M., Barros, L., Ferreira, I. C. F. R., \& Oliveira, M. B. P. P. (2016). Phenolic profile and antioxidant activity of Coleostephus myconis (L.) Rchb.f.: An underexploited and highly disseminated species. Industrial Crops and Products, 89, 45-51. https://doi.org/10.1016/j.indcrop.2016.04.065.

Chauhan, R. S., Kaul, M. K., Shahi, A. K., Kumar, A., Ram, G., \& Tawa, A. (2009) Chemical composition of essential oils in Mentha spicata L. accession [IIIM(J)26] from North-West Himalayan region, India. Industrial Crops and Products, 29(2-3), 654-656. https://doi.org/10.1016/j.indcrop.2008.12.003.

Chrysargyris, A., Panayiotou, C., \& Tzortzakis, N. (2016). Nitrogen and phosphorus levels affected plant growth, essential oil composition and antioxidant status of lavender plant (Lavandula angustifolia Mill.). Industrial Crops and Products, 83, 577-586. https://doi.org/10.1016/j.indcrop.2015.12.067.

Chrysargyris, A., Xylia, P., Botsaris, G., \& Tzortzakis, N. (2017). Antioxidant and antibacterial activities, mineral and essential oil composition of spearmint (Mentha spicata L.) affected by the potassium levels. Industrial Crops and Products, 103(April), 202-212. https://doi.org/10.1016/j.indcrop.2017.04.010.

Cirlini, M., Mena, P., Tassotti, M., Herrlinger, K. A., Nieman, K. M., Dall'Asta, C., \& Rio, D. Del. (2016). Phenolic and volatile composition of a dry spearmint (Mentha spicata L.) extract. Molecules, 21(8), 1-15. https://doi.org/10.3390/molecules21081007.

Clifford, M. N., Knight, S., \& Kuhnert, N. (2005). Discriminating between the six isomers of dicaffeoylquinic acid by LC-MSn. Journal of Agricultural and Food Chemistry, 53(10), 3821-3832. https://doi.org/10.1021/jf050046h.

De Azevedo Neto, A. D., Prisco, J. T., Enéas-Filho, J., Abreu, C. E. B. D., \& Gomes-Filho, E. (2006). Effect of salt stress on antioxidative enzymes and lipid peroxidation in leaves and roots of salt-tolerant and salt-sensitive maize genotypes. Environmental and Experimental Botany, 56(1), 87-94. https://doi.org/10.1016/j.envexpbot.2005.01. 008.

De Carvalho, C. C. C. R. \& Da Fonseca, M. M. R. (2006). Carvone: Why and how should one bother to produce this terpene. Food Chemistry, 95(3), 413-422. https://doi.org/ 10.1016/j.foodchem.2005.01.003.

Dias, M. I., Barros, L., Sousa, M. J., \& Ferreira, I. C. F. R. (2012). Systematic comparison of nutraceuticals and antioxidant potential of cultivated, in vitro cultured and commercial Melissa officinalis samples. Food and Chemical Toxicology, 50(6), 1866-1873. https://doi.org/10.1016/j.fct.2012.03.057.

Emongor, V. E., Chweya, J. A., Keya, S. O., \& Munavu, R. M. (1990). Effect of nitrogen and phosphorus on the essential oil yield and quality of chamomile (Matricaria chamomilla L.) flowers. East African Agricultural and Forestry Journal, 55(4), 261-264. https://doi.org/10.1080/00128325.1990.11663593.

Ferreres, F., Andrade, P., Ferreres, F., Silva, B. M., Andrade, P. B., Seabra, R. M., \& Ferreira, M. A. (2003). Approach to the study of C-glycosyl flavones by ion trap HPLC-PAD-ESI/MS/MS: Application to seeds of quince (Cydonia oblonga). Phytochemical Analysis, 14, 352-359. https://doi.org/10.1002/pca.727.

Foyer, C. H., \& Noctor, G. (2011). Ascorbate and glutathione: The heart of the redox hub. Plant Physiology, 155(1), 2-18. https://doi.org/10.1104/pp.110.167569.

Garlet, T. M. B., \& Dos Santos, O. S. (2008). Nutrient solution and mineral composition of three mint species grown in hydroponic system. Ciência Rural, 38(5), 1233-1239. https://doi.org/10.1590/S0103-84782008000500005.

Gonçalves, S., Moreira, E., Grosso, C., Andrade, P. B., Valentão, P., \& Romano, A. (2017). Phenolic profile, antioxidant activity and enzyme inhibitory activities of extracts from aromatic plants used in Mediterranean diet. Journal of Food Science and Technology, 54(1), 219-227. https://doi.org/10.1007/s13197-016-2453-z.

Guil, J. L., Torija, M. E., Giménez, J. J., Rodríguez-García, I., \& Himénez, A. (1996) Oxalic acid and calcium determination in wild edible plants. Journal of Agricultural and Food Chemistry, 44(7), 1821-1823. https://doi.org/10.1021/jf950472a.

Gülçin, I., Elias, R., Gepdiremen, A., \& Boyer, L. (2006). Antioxidant activity of lignans from fringe tree (Chionanthus virginicus L.). European Food Research and Technology, 223, 759-767. https://doi.org/10.1007/s00217-006-0265-5.

Jiang, M., \& Zhang, J. (2002). Involvement of plasma-membrane NADPH oxidase in 
abscisic acid- and water stress-induced antioxidant defense in leaves of maize seed lings. Planta, 215(6), 1022-1030. https://doi.org/10.1007/s00425-002-0829-y.

Kivilompolo, M., \& Hyötyläinen, T. (2007). Comprehensive two-dimensional liquid chromatography in analysis of Lamiaceae herbs: Characterisation and quantification of antioxidant phenolic acids. Journal of Chromatography A, 1145(1-2), 155-164. https://doi.org/10.1016/j.chroma.2007.01.090.

Loreto, F., \& Velikova, V. (2001). Isoprene produced by leaves protects the photosynthetic apparatus against ozone damage, quenches ozone products, and reduces lipid peroxidation of cellular membranes. Plant Physiology, 127(4), 1781-1787. https://doi. org/10.1104/pp.010497.

Narasimhamoorthy, B., Zhao, L. Q., Liu, X., Yang, W., \& Greaves, J. A. (2015). Differences in the chemotype of two native spearmint clonal lines selected for rosmarinic acid accumulation in comparison to commercially grown native spearmint. Industrial Crops and Products, 63, 87-91. https://doi.org/10.1016/j.indcrop.2014.10.044.

Nell, M., Vötsch, M., Vierheilig, H., Steinkellner, S., Zitterl-Eglseer, K., Franz, C., \& Novak, J. (2009). Effect of phosphorus uptake on growth and secondary metabolites of garden sage (Salvia officinalis L.). Journal of the Science of Food and Agriculture, 89(6), 1090-1096. https://doi.org/10.1002/jsfa.3561.

Nozad, M., Khojastehpour, M., Tabasizadeh, M., Azizi, M., Miraei Ashtiani, S. H., \& Salarikia, A. (2016). Characterization of hot-air drying and infrared drying of spearmint (Mentha spicata L.) leaves. Journal of Food Measurement and Characterization, 10(3), 466-473. https://doi.org/10.1007/s11694-016-9325-0.

Pasternak, T., Rudas, V., Potters, G., \& Jansen, M. A. K. (2005). Morphogenic effects of abiotic stress: Reorientation of growth in Arabidopsis thaliana seedlings. Environmental and Experimental Botany, 53(3), 299-314. https://doi.org/10.1016/j.envexpbot. 2004.04.009.

Pereira, C., Barros, L., Carvalho, A. M., \& Ferreira, I. C. F. R. (2013). Use of UFLC-PDA for the analysis of organic acids in thirty-five species of food and medicinal plants. Food Analytical Methods, 6(5), 1337-1344. https://doi.org/10.1007/s12161-012-9548-6.

Pereira, E., Pimenta, A. I., Calhelha, R. C., Antonio, A. L., Verde, S. C., Barros, L., Ferreira, I. C. F. R. (2016). Effects of gamma irradiation on cytotoxicity and phenolic compounds of Thymus vulgaris L. and Mentha x piperita L. LWT - Food Science and Technology, 71, 370-377. https://doi.org/10.1016/j.lwt.2016.04.004.

Ramezani, S., Rezaei, M. R. \& Sotoudehnia, P. (2009). Improved growth, yield and essential oil content of basil grown under different levels of phosphorus sprays in the field. Journal of Applied Biological Sciences, 3(2), 96-101.

Reich, P. B., Oleksyn, J., \& Wright, I. J. (2009). Leaf phosphorus influences the photosynthesis-nitrogen relation: A cross-biome analysis of 314 species. Oecologia, 160(2), 207-212. https://doi.org/10.1007/s00442-009-1291-3.

Richardson, A. D., Duigan, S. P., Berlyn, G. P., \& Richardson, A. D. (2002). An evaluation of noninvasive methods to estimate foliar chlorophyll content, 185-194.

Rita, I., Pereira, C., Barros, L., Santos-Buelga, C., \& Ferreira, I. C. F. R. (2016). Mentha spicata L. infusions as sources of antioxidant phenolic compounds: Emerging reserve lots with special harvest requirements. Food Funct. 7(10), 4188-4192. https://doi. org/10.1039/C6FO00841K.

Ruberto, G., Baratta, M. T., Dean, S. G., \& Dorman, H. J. D. (2000). Antioxidant and antimicrobial activity of Foeniculum vulgare and Crithmum maritimum essential oils. Planta Medica, 66(8), 687-693.

Sanchez, C. (2016). Phosphorus. In A. V. Barker, \& D. J. Pilbeam (Eds.). Handbook of plant nutrition (pp. 51-90). Boca Raton: Taylor \& Francis.

Scherer, R., Lemos, M. F., Lemos, M. F., Martinelli, G. C., Martins, J. D. L., \& da Silva, A. G. (2013). Antioxidant and antibacterial activities and composition of Brazilian spearmint (Mentha spicata L.). Industrial Crops and Products, 50, 408-413. https://doi. org/10.1016/j.indcrop.2013.07.007.

Sfara, V., Zerba, E., \& Alzogaray, R. (2009). Fumigant insecticidal activity and repellent effect of five essential oils and seven monoterpenes on first-instar nymphs of Rhodnius prolixus. Journal of Medical Entomology, 46(3), 511-515.

Trivino, M. G., \& Johnson, C. B. (2000). Season has a major effect on the essential oil yield response to nutrient supply in Origanum majorana. Journal of Horticultural Science and Biotechnology, 75(5), 520-527. https://doi.org/10.1080/14620316.2000.11511278.

Wojdyło, A., Oszmiański, J., \& Czemerys, R. (2007). Antioxidant activity and phenolic compounds in 32 selected herbs. Food Chemistry, 105(3), 940-949. https://doi.org/ 10.1016/j.foodchem.2007.04.038

Yeşil, M., \& Kara, K. (2016). The effects of different nitrogen and phosphorus doses on essential oil components of some Mentha genotypes. Turkish Journal of Agriculture and Forestry, 40, 882-893. https://doi.org/10.3906/tar-1604-4.

Zhao, D., Oosterhuis, D. M., \& Bednarz, C. W. (2001). Influence of potassium deficiency on photosynthesis, chlorophyll content, and chloroplast ultrastructure of cotton plants. Photosynthetica. https://doi.org/10.1023/A:1012404204910. 